\title{
Resolving Rotational Stacking Disorder and Electronic Level Alignment in a 2D Oligothiophene-Based Lead lodide Perovskite
}

\author{
Manoj K. Jana, ${ }^{\dagger}$ Chi Liu, ${ }^{\ddagger}$ Sven Lidin, ${ }^{\S}$ David J. Dirkes, $"$ Wei You, ${ }^{\prime}$ Volker Blum, ${ }^{\dagger, \$(0)}$ \\ and David B. Mitzi*,+\$0 \\ ${ }^{\dagger}$ Department of Mechanical Engineering and Materials Science, Duke University, Durham, North Carolina 27708, United States \\ ${ }^{\ddagger}$ Department of Chemistry, Duke University, Durham, North Carolina 27708, United States \\ ${ }^{\S}$ Centre for Analysis and Synthesis, Lund University, Lund 22100 , Sweden \\ "Department of Chemistry, University of North Carolina at Chapel Hill, Chapel Hill, North Carolina 27599, United States
}

\begin{abstract}
Two-dimensional (2D) hybrid organic-inorganic perovskites (HOIPs) represent diverse quantum well heterostructures composed of alternating inorganic and organic layers. While 2D HOIPs are nominally periodic in three dimensions for X-ray scattering, the inorganic layers can orient quasi-randomly, leading to rotational stacking disorder (RSD). RSD manifests as poorly resolved, diffuse X-ray scattering along the stacking direction, limiting the structural description to an apparently disordered subcell. However, local ordering preferences can still exist between adjacent unit cells and can considerably impact the properties, particularly the electronic structure. Here, we elucidate RSD and determine the preferred local ordering in the $2 \mathrm{D}[\mathrm{AE} 2 \mathrm{~T}] \mathrm{PbI}_{4} \mathrm{HOIP}$

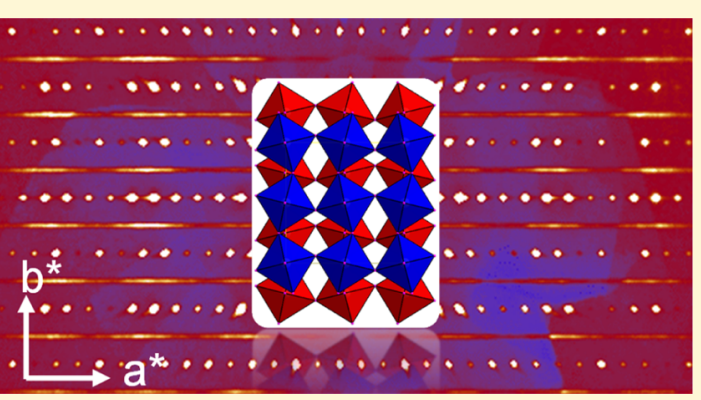

(AE2T: 5,5'-bis(ethylammonium)-[2,2'-bithiophene]). We use first-principles calculations to determine energy differences between a set of systematically generated supercells with different order patterns. We show that interlayer ordering tendencies are weak, explaining the observed RSD in terms of differing in-plane rotation of $\mathrm{PbI}_{6}$ octahedra in neighboring inorganic planes. In contrast, the ordering preference within a given organic layer is strong, favoring a herringbone-type arrangement of adjacent AE2T cations. The calculated electronic level alignments of proximal organic and inorganic frontier orbitals in the valence band vary significantly with the local arrangement of AE2T cations; only the most stable AE2T configuration leads to an interfacial type-Ib band alignment consistent with observed optical properties. The present study underscores the importance of resolving local structure arrangements in 2D HOIPs for reliable structure-property prediction.
\end{abstract}

\section{INTRODUCTION}

Two-dimensional (2D) hybrid organic-inorganic perovskites (HOIPs) are optoelectronic materials exhibiting promise in a broad range of applications, from light-emitting diodes and transistors to spintronics, and are currently in the spotlight of perovskite research owing to several desirable characteristics, including chemical stability and excellent photophysical properties. $^{1-14} 2 \mathrm{D}$ HOIPs are natural quantum well (QW) superlattices (general formula $\mathrm{A}_{2} \mathrm{MX}_{4}$ or $\left.\mathrm{AMX}_{4}\right)^{11,12,15}$ formed by bottom-up self-assembly of semiconducting $\left[\mathrm{MX}_{4}\right]^{2-}$ metal halide perovskite layers alternating with layers of organic $\mathrm{A}^{+}$or $\mathrm{A}^{2+}$ spacer cations. The optical properties in $2 \mathrm{D}$ HOIPs are often governed by the formation of excitons in the perovskite layers. The effects of the excitons are observed even at room temperature due in part to dielectric confinement because of the much lower effective dielectric constant of the organic layers compared with that of the perovskite layers, thereby enhancing the Coulomb interaction and hence the binding energy of the electron-hole pair. ${ }^{16,17}$ The ensuing properties render 2D HOIPs especially attractive for fundamental studies as well as efficient room-temperature optoelectronic applications. ${ }^{10,18-21}$

In contrast to the three-dimensional (3D) analogues, $2 \mathrm{D}$ HOIPs offer wide tunability of optical and electronic properties via tweaking the chemical nature of the organic spacer cation, $^{15,22-27}$ as well as dimensionality and compositional engineering of metal halide layers. ${ }^{28}$ Owing to a plethora of spacer cations of varying length, including aromatic and divalent cations that can be incorporated in between the metal halide layers, 2D HOIPs feature a high structural diversity (broadly classified as $<100>-,<110>-$, and $<111>$-oriented perovskites), as opposed to the limited compositional space accessible for the $3 \mathrm{D} \mathrm{AMX}_{3}\left(\mathrm{~A}=\mathrm{CH}_{3} \mathrm{NH}_{3}{ }^{+}\right.$and $[\mathrm{HC}$ $\left.\left(\mathrm{NH}_{2}\right)_{2}\right]^{+} ; \mathrm{M}=\mathrm{Pb}^{2+}, \mathrm{Sn}^{2+}, \mathrm{Ge}^{2+} ; \mathrm{X}=\mathrm{Cl}^{-}, \mathrm{Br}^{-}$, and $\left.\mathrm{I}^{-}\right)$ analogues. ${ }^{10,29-31}$ Besides serving as templating scaffolds, organic spacer cations can impact the overall optoelectronic properties of $2 \mathrm{D}$ HOIPs by affecting, for example, the metal

Received: August 8, 2019

Revised: $\quad$ September 23, 2019

Published: September 24, 2019 
halide octahedra connectivity/tilting, in-plane/out-of-plane distortions, and perovskite layer orientation, all of which are important factors determining the electronic structure in $2 \mathrm{D}$ HOIPs. ${ }^{29,32,33}$ A comprehensive understanding of the atomic structure is, therefore, indispensable for rationalizing structure-property correlations.

The organic spacer cations hold the metal halide perovskite layers together primarily through hydrogen bonding and electrostatic interactions of their terminal tethering groups (usually with ammonium heads) with the nearest halide atoms $^{34}$ (see Figure S1). Considerable flexibility of the tethering groups can allow the inorganic layers to rotate with respect to one another, giving rise to a rotational stacking disorder (RSD). Though previously not examined in depth, RSD has been observed in a few 2D HOIPs such as (3fluorophenethylammonium) ${ }_{2} \mathrm{SnI}_{4}{ }^{35}$ (2-fluorophenethylammonium $)_{2} \mathrm{SnI}_{4},{ }^{35}[\mathrm{AE} 4 \mathrm{~T}] \mathrm{PbBr}_{4},{ }^{23}[\mathrm{AE} 4 \mathrm{~T}] \mathrm{Bi}_{2 / 3} \square_{1 / 3} \mathrm{I}_{4}(\square=$ vacancy), ${ }^{36}$ manifesting as a weak superstructure ${ }^{35}$ or, in some cases, a diffuse X-ray scattering (DXS) because of random stacking with little or no correlation between adjacent layers. $^{36,37}$ The last feature is the archetypic prerequisite for an order-disorder (OD) structure with equivalent layers, which allows for the coexistence of different polytypes within a single compound. ${ }^{38}$ Rotational stacking faults are prevalent in van der Waals layered solids such as few-layer $\mathrm{MoS}_{2}{ }^{39}$ or graphene $\mathrm{e}^{40}$ with several possible rotational offsets between adjacent layers. In contrast, the RSD in the 2D HOIP class considered here is more restricted in that it only allows for specific, discrete rotations and translations. Analogous types of RSD are realized, for instance, in low-energy face-centered cubic/ hexagonal-closed packing stacking faults in some materials or in the well-known polytypism of $\mathrm{SiC}^{41,42}$ One instance where RSD is inevitably encountered is the class of $2 \mathrm{D}\langle 100\rangle$ oriented metal halide HOIPs with oligothiophene-based divalent spacer cations. ${ }^{23,36,37}$ These 2 D HOIPs constitute an important class of tunable semiconductors where the proximity of inorganic and organic frontier orbitals serves as a knob to tweak the relative band alignments (e.g., by varying the metal/ halide ions and/or length of the oligothiophene) and thereby to control the associated optical excitations and exciton/carrier dynamics across the organic-inorganic interface in a highly tunable fashion. ${ }^{15,43}$ Despite mentioning DXS, the detailed origins of RSD in these and other related HOIPs and its impact on electronic properties are generally ignored.

In this paper, we explore RSD in $2 \mathrm{D}$ HOIPs using the specific example of an oligothiophene-based 2D HOIP, that is, [AE2T $] \mathrm{PbI}_{4}$ (AE2T: 5,5'-diylbis(amino-ethyl)-[2,2' -bithiophene]) (Figure 1a). This compound exhibits poorly resolved DXS along the layer stacking direction because of RSD, as evident from X-ray diffraction. Crystallographic modeling of RSD in 2D HOIPs is, however, not trivial. The description of the crystal structure is limited to a subcell approximation, where different local orientations of organic and inorganic components are modeled as disordered superpositions, that is, the subcell displays disorder that presents atoms within the unit cell at all possible positions that are consistent with the stacking disorder. While this type of model captures the subcell crystallography, it fails to provide information on the degree of local order, i.e., correlations between the orientation of different structural units that are close to one another. Local ordering beyond the crystallographic subcell might occur both in-plane (e.g., between adjacent molecules) and out-of-plane (i.e., rotational correlations between either organic or inorganic

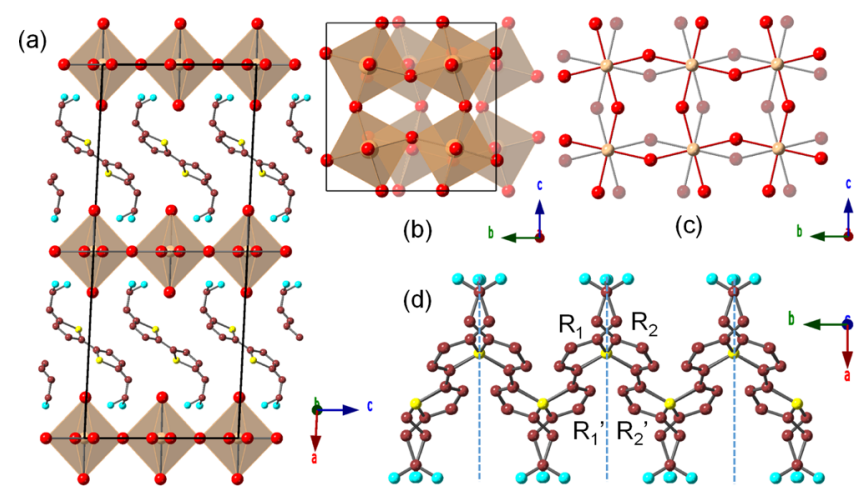

Figure 1. (a) Schematic showing the $2 \mathrm{D}\langle 100\rangle$-oriented layered structure of $[\mathrm{AE} 2 \mathrm{~T}] \mathrm{PbI}_{4}$ in the $1_{\mathrm{b}} \times 2_{\mathrm{c}}$ disordered subcell model $(b=$ $6.18 \AA \times c=12.25 \AA$ ) of the $C 2 / c$ space group. $\mathrm{Pb}, \mathrm{I}, \mathrm{C}, \mathrm{S}$, and $\mathrm{N}$ atoms are denoted by gold, red, brown, yellow, and blue spheres, respectively. (b) Unit cell viewed down the $a$-axis showing the top and middle inorganic layers offset by the lattice C-centering vector (1/ $2,1 / 2,0)$. Only one set of disordered bridging iodine atoms are shown for clarity. (c) View of two distorted frameworks of interconnected equatorial $\mathrm{Pb}-\mathrm{I}-\mathrm{Pb}$ bonds superimposed to give rise to two sets of half-occupied equatorial iodine atoms (denoted by solid and shaded red spheres) within each inorganic layer. (d) View of AE2T cations disordered across the $c$-glide plane (marked as dotted lines) extending as polymeric ribbons along the $b$-axis.

planes) or both. Using first-principles calculations, we show here that the local order preference in $[\mathrm{AE} 2 \mathrm{~T}] \mathrm{PbI}_{4}$ impacts the predicted qualitative electronic level alignment between the organic and inorganic components and must, therefore, be resolved in order to fully understand electronic and optical properties.

In order to understand the origins of RSD and its impact on the crystal and electronic structures of $[\mathrm{AE} 2 \mathrm{~T}] \mathrm{PbI}_{4}$, we have systematically generated a complete set of possible ordered supercells with various configurations of organic-inorganic layers compatible with the experimentally derived disordered subcell solution and evaluated them using density functional theory (DFT). Each inorganic perovskite layer features one of two unique orientations based on rotations of lead iodide octahedra (Figure 1c). AE2T ethylammonium tails are flexible enough to impart rotational freedom to the perovskite layer to switch between these two orientations (Figures 1c and S1), providing no strong preference for specific ordering from one inorganic layer to the next and thereby leading to RSD. Likewise, energetically equivalent AE2T configurations also contribute to disorder, although not in as significant a fashion as the inorganic framework in the context of DXS. However, the packing configuration of AE2T cations within a given organic layer has an important effect on the total energy and energy alignment of organic- and inorganic-derived frontier orbitals across the ordered supercells investigated. As we will show below, the energetically most stable structure adopts a herringbone-type AE2T packing within each organic layer, leading to a type-Ib band alignment at the organic-inorganic interface, consistent with the observed optical behavior of $[\mathrm{AE} 2 \mathrm{~T}] \mathrm{PbI}_{4}{ }^{43}$ Our work emphasizes the importance of rationally addressing stacking disorder and resolving local ordering preferences in 2D HOIPs for reliable property prediction, especially for HOIPs with the complex electronic structure (i.e., with energetically close organic and inorganic band-edge states, as for the present oligothiophene-based systems). 


\section{METHODS}

Synthesis of [AE2T] $\mathrm{Pbl}_{4}$ Single Crystals. The AE2T·HI salt was synthesized in a multistep reaction starting from thiophene-2ethylamine, as described in the Supporting Information of our previous work. ${ }^{37}$ Single crystals of $[\mathrm{AE} 2 \mathrm{~T}] \mathrm{PbI}_{4}(\sim 80 \%$ yield with respect to $\mathrm{Pb}$ ) were grown by slowly cooling a solution of stoichiometric amounts of $\mathrm{PbI}_{2}(3.7 \mathrm{mg})$ and AE2T.HI $(4 \mathrm{mg})$ in 2 $\mathrm{mL}$ of aqueous $\mathrm{HI}$ ( $57 \mathrm{wt} \%$ in $\mathrm{H}_{2} \mathrm{O}$, stabilized) and $0.6 \mathrm{~mL}$ of dimethylformamide in $\mathrm{N}_{2}$ atmosphere from $105{ }^{\circ} \mathrm{C}$ to room temperature over $60 \mathrm{~h}$. The as-obtained small red crystals were filtered, washed with copious amounts of diethyl ether, dried under vacuum, and stored in an $\mathrm{N}_{2}$-filled dry box for further characterization.

Single-Crystal X-ray Diffraction. Room-temperature singlecrystal X-ray diffraction (SC-XRD) was performed on a Bruker APEX II CCD diffractometer operating at $50 \mathrm{kV}$ and $30 \mathrm{~mA}$ using Mo $\mathrm{K} \alpha$ radiation $(\lambda=0.710 \AA)$. A half-sphere of diffraction data was collected with a frame width, an exposure time per frame, and a detector distance of $0.5^{\circ}, 90 \mathrm{~s}$, and $50 \mathrm{~cm}$, respectively. Data integration was performed using the SAINT program. Multiscan empirical absorption correction was applied to the intensity data using the SADABS program. Space groups were determined using the XPREP program. All the above programs are implemented within the Bruker APEX 3 suite. Structure solution was obtained by direct methods using the SHELXS program and refined using the leastsquares method by employing the SHELXL program within the Olex ${ }^{2}$ software. ${ }^{44}$ Single-crystal data of $[\mathrm{AE} 2 \mathrm{~T}] \mathrm{PbI}_{4}$ and refinement results are presented in Table S1.

Computational Methods. All first-principles DFT calculations were performed using the all-electron numeric atom-centered orbital code FHI-aims ${ }^{45-49}$ and the ELSI infrastructure ${ }^{50}$ and ELPA eigenvalue solver ${ }^{51}$ for massively parallel simulations. Full relaxation of lattice parameters and in-cell atomic coordinates were performed for all crystal geometries, relying on van der Waals-corrected semilocal DFT. Specifically, the Perdew-Burke-Ernzerhof (PBE) exchange-

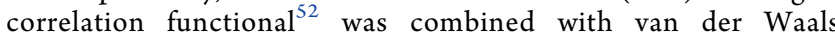
interactions in the Tkatchenko-Scheffler (TS) pairwise dispersion scheme. ${ }^{53}$ FHI-aims' "tight" numerical defaults were employed, and $k$ point grids of $1 \times 2 \times 2$ were used to sample the Brillouin zone. For electronic properties (energy band structures), the Heyd-ScuseriaErnzerhof (HSE06) hybrid density functional ${ }^{54}$ plus perturbative spin-orbit coupling (SOC) $)^{55}$ was employed to predict frontier orbitals and band structure properties. For the band structure calculations, FHI-aims' "intermediate" numerical settings were employed, and $3 \times 3 \times 3 k$-point grids were used to sample the Brillouin zone. A numerical comparison of two particular band structures calculated using "intermediate" versus "tight" settings reveals only negligible differences (see Figure S4 in the Supporting Information), demonstrating the reliability of the "intermediate" settings for this and other calculated energy band structures presented in this work. To retain consistency for the comparison of the energy band structures of different materials, the HSE06 exchange mixing parameter $\alpha$ was fixed at $25 \%$, and the screening parameter $\omega$ was fixed at $0.11 \mathrm{bohr}^{-1}$.

\section{RESULTS AND DISCUSSION}

General Structural Characteristics and Subcell Model. $[\mathrm{AE} 2 \mathrm{~T}] \mathrm{PbI}_{4}$ crystallizes as a $\langle 100\rangle$-oriented layered perovskite, where the inorganic $\left[\mathrm{PbI}_{4}\right]^{2-}$ layers of corner-sharing $\mathrm{PbI}_{6}$ octahedra are separated by layers of AE2T cations (Figure 1a). Charge-coupled device (CCD) frames from SC-XRD at $298 \mathrm{~K}$ reveal well-defined X-ray reflections coexisting with diffuse reflections, as shown in Figure 2a. The former can be indexed to a monoclinic subcell $(\mathrm{C} 2 / \mathrm{c}$ space group) with lattice parameters $a=29.7184(13) \AA, b=6.1756(3) \AA, c=$ 12.2470(6) $\AA$ and $\beta=92.7847(10)^{\circ}$ (see Table S1), whereas the latter are characteristic of RSD, resulting in doubled $b$ and/or $a$-axes. A precession image of the reciprocal lattice
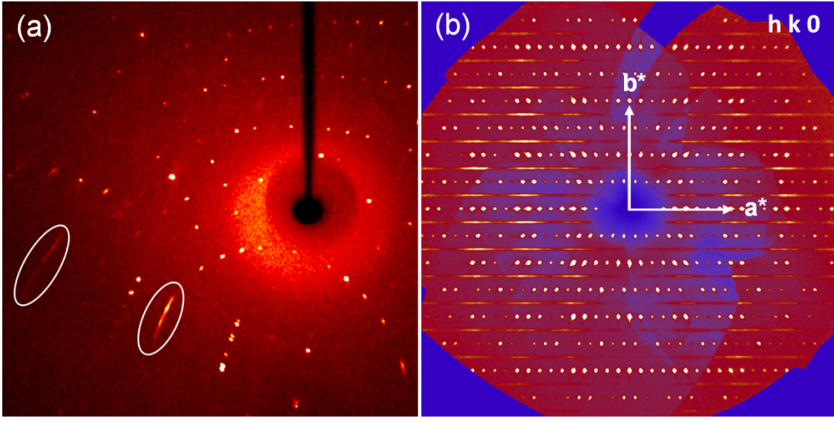

Figure 2. (a) Typical CCD image of XRD showing the coexistence of well-defined reflections and streaklike, diffuse reflections (highlighted by white ellipses). (b) Precession image of the reciprocal lattice, reconstructed along $h k 0$, revealing diffuse streaks continuous along the $a^{*}$ direction with the loci $(x, 1 / 2+k, l)$.

reconstructed along $h k 0$ (Figure $2 \mathrm{~b}$ ) reveals poorly resolved diffuse scattering with a streaklike profile that is nearly continuous along the $a^{*}$-direction in reciprocal space. ${ }^{23,35,36}$ RSD (implying disorder) is not trivial to model crystallographically, thus complicating an accurate structure description and subsequent electronic structure modeling. Such DXS has also been observed in other 2D HOIPs (as presented in the Introduction). Although not specifically discussed in the context of RSD, their crystal structures, as with the present $[\mathrm{AE} 2 \mathrm{~T}] \mathrm{PbI}_{4}$, could only be refined using a subcell. Attempts to employ the larger supercell in each case have not yielded meaningful results during the structure refinement process, owing to the diffuse nature of the associated X-ray reflections defining the larger cell.

The above monoclinic $\mathrm{C} 2 / \mathrm{c}$ subcell model represents an average structure for $[\mathrm{AE} 2 \mathrm{~T}] \mathrm{PbI}_{4}$. Within this model, successive inorganic layers are offset by the lattice $C$-centering vector $(1 / 2,1 / 2,0)$, rendering them out-of-phase along the $b$ direction. Along the $c$-direction, they are offset by the monoclinic angle of $\beta=92.7847(10)^{\circ}$ (Figure 1b). Each inorganic layer is composed of $\mathrm{PbI}_{6}$ octahedra that share vertices in the $b-c$ plane, and each $\mathrm{PbI}_{6}$ octahedron is rotated around the $\mathrm{I}-\mathrm{Pb}-\mathrm{I}$ axis perpendicular to the $b-c$ plane such that $\mathrm{Pb}-\mathrm{I}-\mathrm{Pb}$ equatorial bond angles significantly deviate from $180^{\circ}$ [that is, $152.29(9)^{\circ}, 152.40(9)^{\circ}$, and $153.55(6)^{\circ}$ ]. As a result of these octahedron rotations (or inorganic lattice distortions), any given square of four adjacent corner-sharing $\mathrm{PbI}_{6}$ octahedra, defined by four interconnected equatorial $\mathrm{Pb}-$ $\mathrm{I}-\mathrm{Pb}$ bonds, must be "pulled-in" and "pushed-out" along $b$ and $c$-directions, respectively, or vice versa. The distortion pattern of each square fixes the nearest-neighboring square of octahedra to be in the opposite distortion pattern (see Figure 1c); otherwise, unphysically short I $\cdots I$ bond distances or incorrect out-of-plane octahedral tilts would result. ${ }^{15}$ Consequently, the distortions within the $b-c$ plane (and therefore the lead iodide layer itself) must be fully ordered. This requires a unit cell with $b$ and $c$ lattice parameters that allow fitting $2 \times$ 2 copies of the original $\left[\mathrm{PbI}_{6}\right]$ octahedron in the $b-c$ plane. However, in the $\sim 1_{\mathrm{b}} \times 2_{\mathrm{c}}$ subcell model $(6.18 \AA \times 12.25 \AA)$ considered above, the inorganic layer exhibits an unreconcilable disorder: the two unique patterns of square distortion are superimposed with equal probability, resulting in an apparent disorder of equatorial iodine atoms over two symmetry-related sites with a chemical occupancy of 0.5 for each site (Figure 1c). To capture the ordering in the $b-c$ plane (which must exist in 
the actual structure within each perovskite layer), the $b$ parameter must be doubled, that is, a $2_{b} \times 2_{c}$ unit cell must be used.

The reciprocal lattice precession image along an $h k 0$ section (Figure 2b) displays diffuse streaks nearly continuous along the $a^{*}$-direction with the loci $(x, 1 / 2+k, l)$. This indicates that the $b$-direction is indeed doubled, but the ordering in one $b-c$ plane is not transferred to the surrounding layers along the $a$ direction. This is indeed the archetypic prerequisite for an OD structure, which allows for the coexistence of different polytypes within a single compound. ${ }^{38}$ In terms of local ordering, this means that the energy difference between two distinct stacking sequences is small. Typically, any two-layer stacking arrangements are equivalent, while three-layer stackings are distinct. A simple comparison is to the hexagonal and cubic close packings. We normally denote hexagonal close packing as $\mathrm{ABABAB}$ and cubic as $\mathrm{ABCABC}$; however, all twolayer sequences $\mathrm{AB}, \mathrm{AC}$, and $\mathrm{BC}$ are in fact equivalent, and so, hexagonal close packing may equally well be denoted as ACACAC or BCBCBC. The difference only comes into play with the addition of a third layer. $\mathrm{ABA}$ is distinct from $\mathrm{ABC}$ because of the relationship between the third layer and the second and the first. Similarly, more complex layer stackings become distinct only with the addition of more layers; for example, the five-layer samarium stacking $A B C A B A B C A B$ only becomes different from the cubic stacking by the addition of the sixth layer. The energetics of the system determines the frequency of stacking variations. If there is no energy difference between the sequences $\mathrm{ABCABC}$ and $\mathrm{ABABAB}$, a completely disordered structure is expected with only diffuse scattering, while a strong preference for a specific order leads to at most a few stacking faults and sharper, more well-defined X-ray reflections.

The lack of registry between adjacent inorganic layers in [AE2T $] \mathrm{PbI}_{4}$ due to RSD is possible given the flexibility of the AE2T ethylammonium tethering groups connecting the inorganic layers via hydrogen bonding interactions with the nearest iodine atoms (see Figure S1). In the organic layer, all the carbon atoms (except the terminal one attached to the ammonium group) and nitrogen atoms of the AE2T cation are disordered across the $c$-glide plane (Figure 1d). The asymmetric unit contains two distinct thiophene rings (denoted as $R_{1}$ and $R_{2}$ ), and the other half of each bithiophene moiety (i.e., $R_{1}^{\prime}$ and $R_{2}^{\prime}$ ) is generated by an inversion symmetry (Figure 1d). The two thiophene rings within each AE2T moiety are coplanar and adopt an "anti" configuration (Figure 1c), as observed in neat organic crystals of unsubstituted bithiophene molecules ${ }^{56}$ as well as related hybrids incorporating substituted bithiophene cations. ${ }^{57}$ The apparent disorder for the AE2T cation is, as for the inorganic layer configuration, a consequence of stacking disorder and the choice of subcellthe two half-occupied (i.e., equal probability) configurations of the AE2T backbone in the present $C 2 / c$ subcell $\left(\sim 1_{b} \times 2_{c}\right)$ model become superimposed to extend as ribbons along the $b$ direction (Figure 1c).

Generation of Ordered Supercells. The natural interpretation of the diffuse scattering is that a continuous set of local unit cells is allowed. As a starting point for generating models of these larger ordered cells, the average disordered $\mathrm{C} 2 / \mathrm{c}$ structural solution was used in an augmented cell with the $b$-axis doubled. In this cell, it is possible to generate ordered inorganic layers. If we start from the inorganic layers, there are two of these in the unit cell and, although they are correlated by the $C$-centering in the average unit cell, no such correlation needs to apply locally. There are thus formally four different configurations of the inorganic layers, two for each layer; however, they are all equivalent up to a translation or a reflection. The four configurations of the inorganic layers in the augmented cell with a doubled $b$-axis are shown in Figure 3. The top and bottom halves differ in the

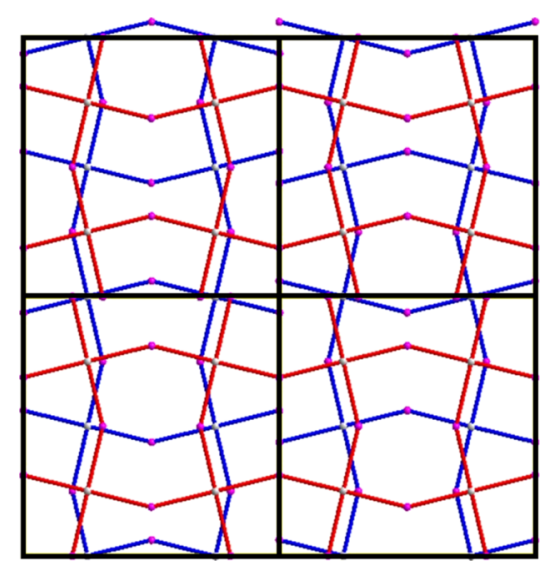

Figure 3. Four possible configurations (bound by four boxes marked in black) for the two inorganic layers in an augmented $2_{\mathrm{b}} \times 2_{\mathrm{c}}$ unit cell. The first and second layers are marked in red and blue, respectively. $\mathrm{Pb}$ and $\mathrm{I}$ atoms in each layer are denoted by white and pink spheres, respectively.

choice of configuration of one layer (red), while the left and right halves differ in the configuration of the other layer (blue). Note that the images to the left are mirror images of those to the right and that the image to the upper left is identical to that on the lower right with respect to a translation of $1 / 2$ in the vertical direction. In a model with two unique inorganic layers, we may, therefore, use a single configuration without any loss of generality.

The repeat along the $a$-axis in the average $C 2 / c$ structure is $29.7 \AA$, and this is the shortest allowed $a$-repeat (i.e., two inorganic layers). The diffuse scattering indicates that a distribution of other longer $a$-repeats is permitted, but the energy differences for these involve correlations across $\sim 30 \AA$, while the ordering within the organic layers and between one organic layer and the next, across an inorganic layer, involves much shorter distances, and consequently, these are assumed to be much more critical. An AE2T molecule may connect equivalent points on successive inorganic layers along the $a$ axis given by the centering vector $(1 / 21 / 40)$ or $(1 / 2-1 / 4$ $0)$, and all molecules in rows along the $b$-axis must be in register because of spatial requirements; however, for rows along the $c$-axis, the orientation may alternate. There are hence four different configurations for a single organic layer: all AE2T molecules leaning left in projection $\mathrm{L}$, all AE2T molecules leaning right in projection $\mathrm{R}$, and two other possible configurations where rows leaning left and right alternate along the $c$-axis (i.e., in a herringbone arrangement). The latter two are distinguished by choice of origin, and the labels $\mathrm{X}^{+}$and $\mathrm{X}^{-}$are used to differentiate between them. For the second organic layer, the possibilities are the same. The configurations $\mathrm{L}$ and $\mathrm{R}$ and the configurations $\mathrm{X}^{+}$and $\mathrm{X}^{-}$are related by reflection symmetry, and we may, therefore, restrict our choice of the first layer to $\mathrm{L}$ and $\mathrm{X}^{+}$and we come up with a full set of eight configurations that may be labeled LL, LR, $\mathrm{LX}^{+}, \mathrm{LX}^{-}$, 
$\mathrm{X}^{+} \mathrm{L}, \mathrm{X}^{+} \mathrm{R}, \mathrm{X}^{+} \mathrm{X}^{+}, \mathrm{X}^{+} \mathrm{X}^{-}$(Figure 4 ). In the augmented average cell, each of these configurations can be identified and separated so that fully ordered models may be generated.

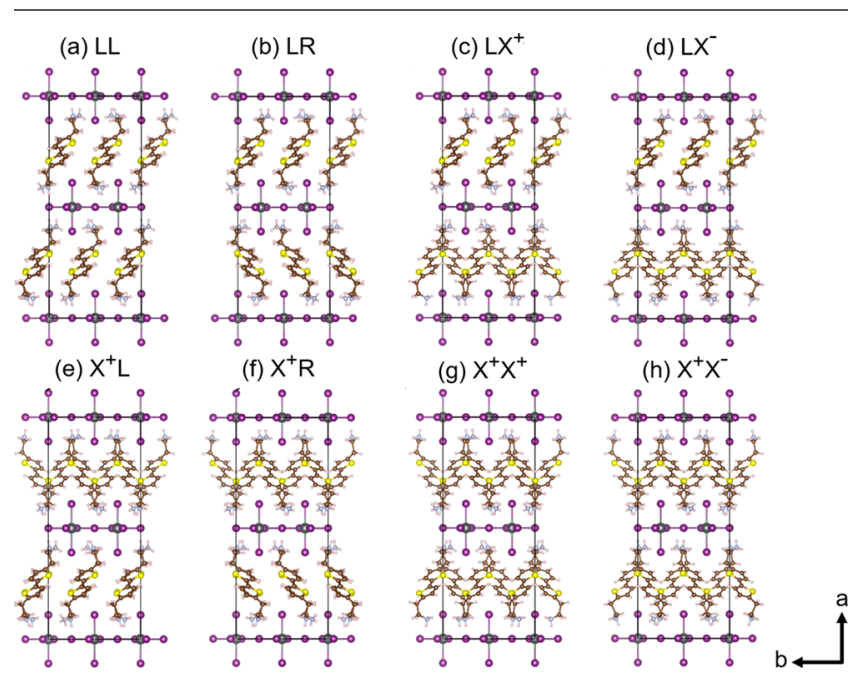

Figure 4. $(\mathrm{a}-\mathrm{h})$ Eight possible ordered $2_{\mathrm{b}} \times 2_{\mathrm{c}}$ supercell models (viewed down the $c$-axis) of $[\mathrm{AE} 2 \mathrm{~T}] \mathrm{PbI}_{4}$ with different configurations of the AE2T cations. L: AE2T cations are all oriented to the left along the $b$-axis and are in-phase along the $c$-axis; R: AE2T cations are all oriented to the right along the $b$-axis and are in-phase along the $c$-axis; $\mathrm{X}+$ : AE2T cations are all oriented to the right along the $b$-axis and are out-of-phase along the $c$-axis (i.e., oriented to the left); X-: AE2T cations are all oriented to the left along the $b$-axis and are out-of-phase along the $c$-axis (i.e., oriented to the right). The first and second letters in each of the eight labels correspond, respectively, to the upper and lower layers of AE2T cations in the unit cell along the $a$ direction. Dark-gray, purple, yellow, brown, blue, and light-pink spheres denote $\mathrm{Pb}, \mathrm{I}, \mathrm{S}, \mathrm{C}, \mathrm{N}$, and $\mathrm{H}$ atoms, respectively.

It is important to note that the positions of the ammonium groups are given by the configuration of the inorganic layer to which they are bound via hydrogen bonding (see Figure S1). The position of the terminal carbon atom to which the ammonium group is attached is almost invariant with the orientation of either the inorganic or the organic part. This is the hinge that allows independence between the different configurations. The effect of the varying orientations of the rest of the local structure can only be detected as a slightly enhanced anisotropy of the displacement parameter for this carbon atom. The configuration of the organic molecule is, therefore, largely uncorrelated to that of the inorganic layer. The eight models that define ordered structural units that may appear in the average disordered system with RSD were built by identifying modules in the average structure that contain the inorganic part and the ammonium group and other modules that contain the different organic constellations. There is no indication in the X-ray data that allows for distinguishing between the different models for ordering. However, the actual structure will still prefer one or more of the specific types of local order (i.e., the local correlation between relative orientations of nearby molecules). The structural preferences depend on the relative energies of different ordered motifs. Their relevance can, therefore, be assessed by theoretical calculations.

First-Principles Calculations. The eight structural models in Figure 4 were each optimized using the DFT-PBE + TS method, allowing all lattice parameters and in-cell atomic coordinates to relax to the minimum-energy geometry for each structure. The relaxed lattice parameters for all the eight structures are summarized in Table 1 along with their \% deviation from the experimental lattice parameters corresponding to the augmented $C 2 / c$ structure (i.e., with doubled $b$-axis). The PBE + TS energy differences between the eight optimized structures are plotted in Figure 5, where the reference energy value is given by the most stable structure, $\mathrm{X}^{+} \mathrm{X}^{-}$. Both $\mathrm{X}^{+} \mathrm{X}^{+}$ and $\mathrm{X}^{+} \mathrm{X}^{-}$are found to be energetically practically identical (difference of $0.083 \mathrm{meV} /$ atom) with similar optimized lattice

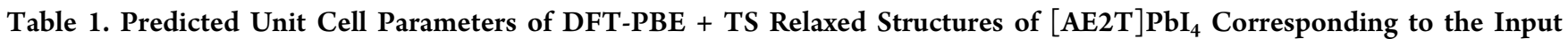
$2 \times 2$ Supercell Models Shown in Figure 4, along with Their \% Deviation with Respect to Experimental Lattice Parameters of the Augmented C2/c Unit Cell

\begin{tabular}{|c|c|c|c|c|c|c|c|c|}
\hline no. & structure & $a$ & $b$ & $c$ & $\alpha$ & $\beta$ & $\gamma$ & $V$ \\
\hline 0 & exp. $(2 \times 2)$ & 29.71 & 12.36 & 12.25 & 90.0 & 92.8 & 90.0 & 4492 \\
\hline \multirow[t]{2}{*}{1} & $\mathrm{LL}$ & 30.30 & 12.30 & 12.16 & 86.8 & 92.2 & 89.2 & 4520 \\
\hline & deviation & $1.97 \%$ & $-0.44 \%$ & $-0.76 \%$ & $-3.50 \%$ & $-0.68 \%$ & $-0.86 \%$ & $0.62 \%$ \\
\hline \multirow[t]{2}{*}{2} & LR & 30.47 & 12.30 & 12.11 & 90.0 & 92.7 & 90.0 & 4534 \\
\hline & deviation & $2.55 \%$ & $-0.44 \%$ & $-1.15 \%$ & $0.00 \%$ & $-0.05 \%$ & $-0.01 \%$ & $0.94 \%$ \\
\hline \multirow[t]{2}{*}{3} & $\mathrm{LX}^{+}$ & 30.30 & 12.28 & 12.11 & 88.7 & 91.7 & 89.3 & 4503 \\
\hline & deviation & $1.99 \%$ & $-0.60 \%$ & $-1.15 \%$ & $-1.50 \%$ & $-1.18 \%$ & $-0.78 \%$ & $0.25 \%$ \\
\hline \multirow[t]{2}{*}{4} & $\mathrm{LX}^{-}$ & 30.28 & 12.28 & 12.12 & 88.7 & 91.7 & 89.4 & 4502 \\
\hline & deviation & $1.90 \%$ & $-0.60 \%$ & $-1.08 \%$ & $-1.50 \%$ & $-1.21 \%$ & $-0.68 \%$ & $0.23 \%$ \\
\hline \multirow[t]{2}{*}{5} & $\mathrm{X}^{+} \mathrm{L}$ & 30.21 & 12.30 & 12.14 & 88.7 & 91.7 & 89.5 & 4505 \\
\hline & deviation & $1.67 \%$ & $-0.49 \%$ & $-0.91 \%$ & $-1.46 \%$ & $-1.17 \%$ & $-0.59 \%$ & $0.29 \%$ \\
\hline \multirow[t]{2}{*}{6} & $\mathrm{X}^{+} \mathrm{R}$ & 30.31 & 12.28 & 12.11 & 91.3 & 91.7 & 90.7 & 4504 \\
\hline & deviation & $2.02 \%$ & $-0.58 \%$ & $-1.17 \%$ & $1.48 \%$ & $-1.15 \%$ & $0.83 \%$ & $0.28 \%$ \\
\hline \multirow[t]{2}{*}{7} & $\mathrm{X}^{+} \mathrm{X}^{-}$ & 30.18 & 12.27 & 12.12 & 90.0 & 91.1 & 90.0 & 4485 \\
\hline & deviation & $1.58 \%$ & $-0.73 \%$ & $-1.08 \%$ & $-0.05 \%$ & $-1.82 \%$ & $0.03 \%$ & $-0.15 \%$ \\
\hline \multirow[t]{2}{*}{8} & $\mathrm{X}^{+} \mathrm{X}^{+}$ & 30.16 & 12.27 & 12.12 & 90.0 & 91.1 & 90.0 & 4486 \\
\hline & deviation & $1.50 \%$ & $-0.67 \%$ & $-1.03 \%$ & $-0.04 \%$ & $-1.81 \%$ & $0.04 \%$ & $-0.13 \%$ \\
\hline \multirow[t]{2}{*}{9} & ${ }^{a} \mathrm{X}^{+} \mathrm{X}^{+}$ & 30.16 & 12.27 & 12.12 & 90.0 & 91.1 & 90.0 & 4486 \\
\hline & deviation & $1.50 \%$ & $-0.67 \%$ & $-1.03 \%$ & $-0.04 \%$ & $-1.81 \%$ & $0.04 \%$ & $-0.13 \%$ \\
\hline
\end{tabular}

${ }^{a} \mathrm{X}^{+} \mathrm{X}^{+}$model with a different configuration of inorganic layers. 


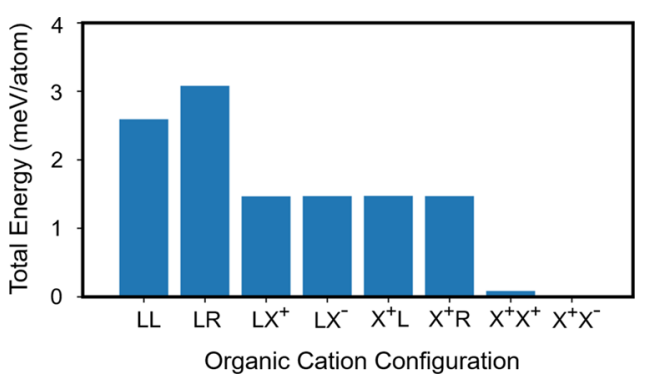

Figure 5. DFT-PBE + TS energy difference per atom of the relaxed $[\mathrm{AE} 2 \mathrm{~T}] \mathrm{PbI}_{4}$ structure for the eight enumerated supercells. The total energy of the $\mathrm{X}^{+} \mathrm{X}^{-}$structure is taken as the reference value, with respect to which all other energy differences are computed. The energy differences ( $\mathrm{meV} /$ atom) are plotted along the $y$-axis for all the eight supercells denoted by the $x$-axis labels.

parameters (see Table 1). The considerably higher total energies for the other structures $(>1 \mathrm{meV} /$ atom, with 312 atoms per structural unit considered) suggest that they are less favored relative to $\mathrm{X}^{+} \mathrm{X}^{+}$and $\mathrm{X}^{+} \mathrm{X}^{-}$(i.e., these other configurations are less likely to play a large role in the experimental RSD structure).

Further evidence for the favorability of the $\mathrm{X}^{+} \mathrm{X}^{+}$or $\mathrm{X}^{+} \mathrm{X}^{-}$ structures stems from a comparison with the neat bithiophene crystal structure $^{56}$ where the bithiophene molecules pack in a herringbone fashion with edge-to-face (tilted-T type) aromatic interactions between adjacent molecules (Figure 6a). The

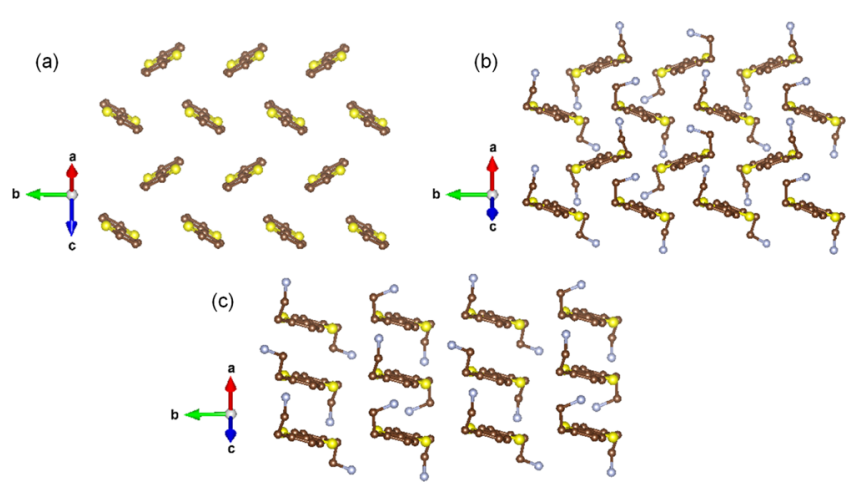

Figure 6. (a) Herringbone arrangement of bithiophene molecules within a single sheet of the pristine $2,2^{\prime}$-bithiophene crystal, (b) herringbone arrangement of AE2T cations within a single organic layer of the $\mathrm{X}^{+} \mathrm{X}^{+}$supercell, and (c) $\beta$-type arrangement of AE2T cations within a single organic layer of the LL supercell, viewed along similar directions. Yellow and brown spheres denote sulfur and carbon atoms, respectively.

herringbone arrangement of AE2T cations in the optimized $\mathrm{X}^{+} \mathrm{X}^{+}\left(\right.$or $\mathrm{X}^{+} \mathrm{X}^{-}$) model as well as in the experimental $\mathrm{C} 2 / \mathrm{c}$ subcell structure (i.e., considering only one-half of the disordered AE2T cations) resembles that in the neat 2,2'bithiophene crystal structure (Figure 6a,b); the respective herringbone angles are slightly different, that is, $42.35^{\circ}$ and $44.32^{\circ}$ compared with the $58.35^{\circ}$ angle in $2,2^{\prime}$-bithiophene crystals. The preferred herringbone arrangement in either case-albeit with slight changes induced by terminal functional groups on the bithiophene backbone and their hydrogen bonding interactions with the inorganic layers-suggests that the $2 \mathrm{D}$ inorganic layers may in part be templated by the AE2T layers, as discussed earlier for the $2 \mathrm{D}[\mathrm{AE} 2 \mathrm{~T}]_{2} \mathrm{AgBiI}_{8} \mathrm{HOIP}^{37}$ In fact, a related asymmetrical, monosubstituted bithiophene cation with a sulfur ethyl ammonium tethering group (i.e., AES2T $\left.=\left(\mathrm{C}_{4} \mathrm{~S}\right)_{2} \mathrm{~S}\left(\mathrm{CH}_{2}\right)_{2} \mathrm{NH}_{3}{ }^{+}\right)$forms a nonperovskite $[\mathrm{AES} 2 \mathrm{~T}]_{4} \mathrm{~Pb}_{3} \mathrm{I}_{10} 1 \mathrm{D}$ hybrid, whereas its bisubstituted symmetrical analogue akin to the AE2T cation here (i.e., BAES2T $\left.=\mathrm{NH}_{3}\left(\mathrm{CH}_{2}\right)_{2} \mathrm{~S}\left(\mathrm{C}_{4} \mathrm{~S}\right)_{2} \mathrm{~S}\left(\mathrm{CH}_{2}\right)_{2} \mathrm{NH}_{3}\right)_{2}^{+}$) forms (BAESBT) $\mathrm{PbI}_{4}$ $2 \mathrm{D}$ HOIP. ${ }^{57}$ This suggests a considerable role of the symmetry or functionalization of the organic cation (i.e., mono vs bisubstitution)-besides hydrogen bonding and aromatic interactions-in the stabilization of $2 \mathrm{D}$ HOIPs over nonperovskite counterparts. It is further noted that [AES2T $]_{4} \mathrm{~Pb}_{3} \mathrm{I}_{10}$ exhibits a herringbone packing of organic cations, while (BAESBT) $\mathrm{PbI}_{4}$ exhibits a $\beta$-type packing, where the adjacent organic molecules within a single organic layer are nearly parallel to one another. ${ }^{57}$ The latter $\beta$-type packing is seen for some of the proposed models with $\mathrm{L}$ or $\mathrm{R}$ configuration of AE2T cations either in one or both the organic layers in the unit cell (Figure 6c). Although herringbone packing is more commonly encountered among pristine oligothiophenes, ${ }^{58} \beta$-type packing also occurs. ${ }^{57}$ For $[\mathrm{AE} 2 \mathrm{~T}] \mathrm{PbI}_{4}$, the preference for herringbone packing (e.g., $\mathrm{X}^{+} \mathrm{X}^{+}$) over $\beta$-type packing (e.g., LL) in Figure $6 \mathrm{~b}, \mathrm{c}$ is definitively validated by the calculated DFT-PBE + TS total energies in Figure 5.

The two energetically equivalent organic configurations, that is, $\mathrm{X}^{+} \mathrm{X}^{+}$and $\mathrm{X}^{+} \mathrm{X}^{-}$, are structurally different, but their very similar total energies suggest that the correlation of molecular orientation across adjacent organic layers is weak at best. Their roughly equivalent likelihood of occurrence can give rise to distinct diffraction and contribute to the observed disorder. However, from the perspective of the X-ray diffraction, the inorganic framework is more important because it contributes more strongly to the $\mathrm{X}$-ray scattering (due to heavy $\mathrm{Pb}$ and $\mathrm{I}$ atoms). Besides the enumeration of possible combinations and orderings of organic and inorganic layers, along with the associated energy differences (which enter the likelihood of finding each configuration), it is also important to consider what effect the configurational preference has on the electronic structure. In the case of $[\mathrm{AE} 2 \mathrm{~T}] \mathrm{PbI}_{4}$, this is a particularly interesting question, given that the conjugated organic cation may play a role in the definition of the frontier orbitals of the valence band.

DFT-HSE06 + SOC-based electronic band structures calculated for all the eight optimized structures are shown in Figure 7 . In the $2 \times 2$ arrangement considered here, all the structures exhibit a direct band gap at the $\Gamma$ point, with values ranging from 1.99 to $2.08 \mathrm{eV}$ among the different structures. As shown in the Supporting Information of ref 37 , the $\mathrm{Pb}-\mathrm{I}$ derived conduction band minimum (CBM) in the smaller $1 \times$ 2 cell would not be at the $\Gamma$ point, that is, the direct gaps shown in Figure 7 may only result from backfolding after expansion to the larger, ordered $2 \times 2$ arrangements shown in Figures 4 and S2 of the present work. In Figure 7, the Pb-, I-, and organic-related states are identified by color according to the projected Mulliken decomposition of the associated states onto different species. We find that the CBM in all the structures is dominated by $\mathrm{Pb}$-derived states with a contribution from I-derived states and the empty flat bands (black) resulting from the AE2T component appear $\sim 1 \mathrm{eV}$ higher than the CBM. On the other hand, the character of the valence band maximum (VBM) is more complicated because of the proximity of inorganic and organic highest occupied molecular orbital (HOMO) levels. The configuration of the AE2T cations also impacts the inorganic framework as evident 
(a)

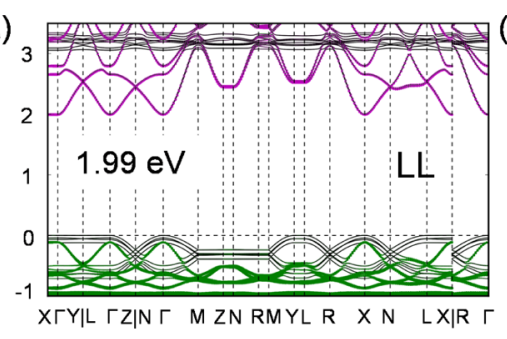

(c)

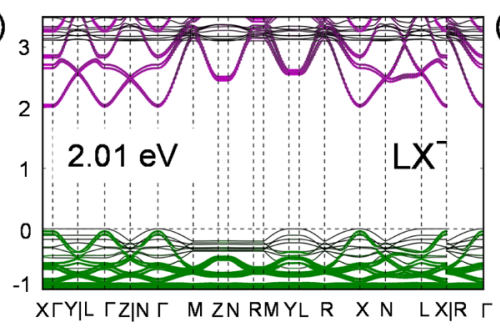

(e)

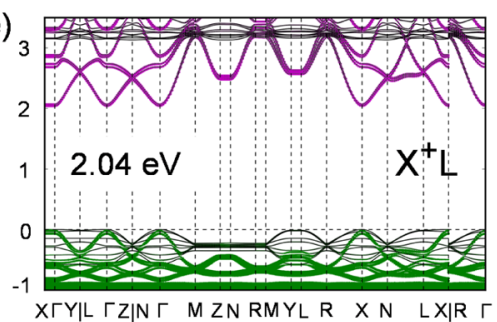

(b)

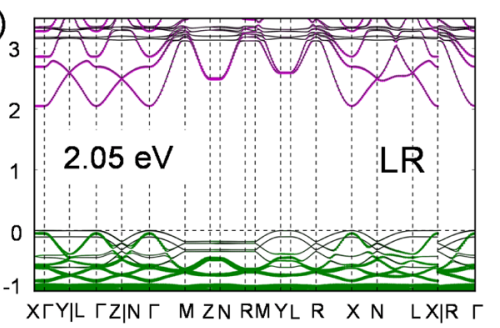

(d)

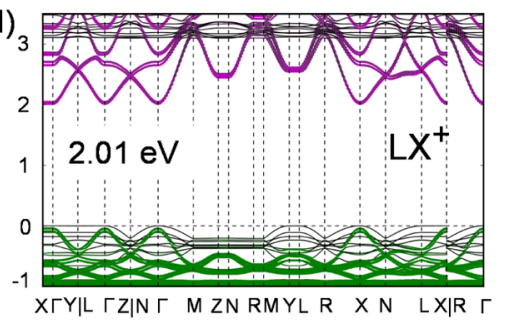

(g)

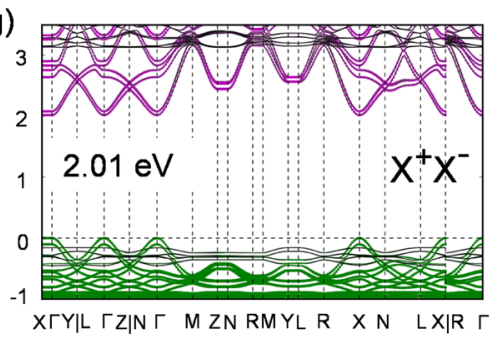

(f)

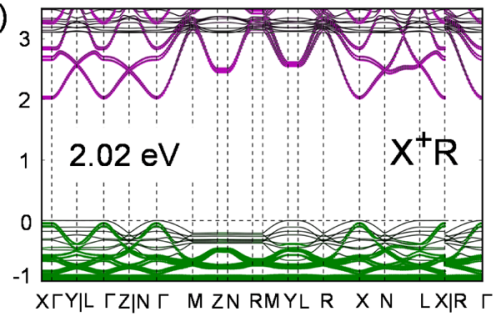

(h)

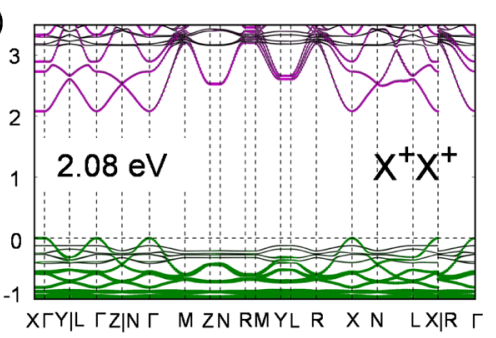

Figure 7. $(\mathrm{a}-\mathrm{h})$ Band structures of the eight enumerated $[\mathrm{AE} 2 \mathrm{~T}] \mathrm{PbI}_{4}$ structures calculated by DFT-HSE06 + SOC, with the states of Pb (magenta) and I (green) identified by projected density of states onto different species. The thin black lines correspond to bands that are mainly derived from the organic moiety. It is noteworthy that the inorganic-derived bands show significantly more dispersion than those derived from the organic species and that the valence band character changes from organic-derived $(a-f)$ to inorganic-derived $(g, h)$ as a function of the organic configuration. The zero for the energy scale in all subplots is set equal to the VBM. For the $\mathrm{X}^{+} \mathrm{X}^{-}$configuration shown in Figure $7 \mathrm{~g}$, the band structure was recalculated using FHI-aims' "tight" settings as shown in Figure S4b, showing very little quantitative and no qualitative changes compared to the FHI-aims' "intermediate" numerical settings used here.

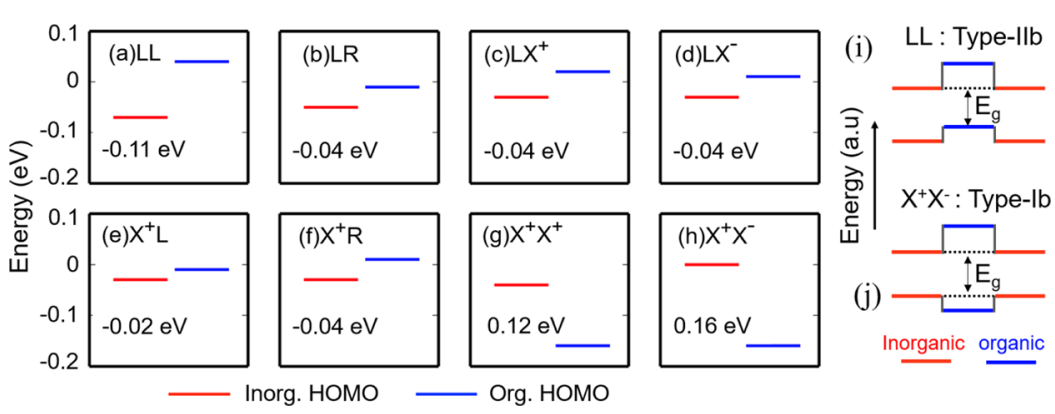

Figure 8. $(\mathrm{a}-\mathrm{h})$ Frontier orbital alignments for inorganic and organic HOMO levels for each of the eight enumerated supercells of $\left[\mathrm{AE}_{2} \mathrm{~T}\right] \mathrm{PbI}$ predicted by the DFT-HSE06 + SOC analysis. The energy levels of different structures are aligned relative to the average of Pb $1 \mathrm{~s}$ energies. Inorganic HOMO for the $\mathrm{X}^{+} \mathrm{X}^{-}$model is set as the reference zero value. The number printed in each subfigure is the difference between the inorganic and organic HOMO energy values, that is, $E_{\text {(inorg. номо) }}-E_{\text {(org. HOMO) }}$. Schematic representation of (i) type-IIb and (j) type-Ib band alignments across the inorganic-organic interface, found for the $\mathrm{LL}$ and $\mathrm{X}^{+} \mathrm{X}^{-}$models, respectively. $E_{\mathrm{g}}$ denotes the band gap.

from the geometry optimization. As a result, the relative alignment of the inorganic and organic HOMO levels is sensitive to the exact atomic structure on which the band structure calculations are based.
In Figure 8, we plot the inorganic and organic HOMO levels of all the eight structures extracted from their respective band structures by projecting DOS onto different species. We find that the inorganic HOMO is higher in energy than the organic 
HOMO for the two most stable structures predicted here, that is, $\mathrm{X}^{+} \mathrm{X}^{+}$and $\mathrm{X}^{+} \mathrm{X}^{-}$. This level alignment is indeed consistent with recent photoluminescence $(\mathrm{PL})$ studies on $[\mathrm{AE} 2 \mathrm{~T}] \mathrm{PbI}_{4}$ thin films, ${ }^{43}$ for which a strong excitonic emission from the $2 \mathrm{D}$ $\left[\mathrm{PbI}_{4}\right]^{2-}$ layers is observed, indicating a type-Ib alignment (as per the definition given in ref 15 ) in which both the CBM and VBM are dominated by the states from the inorganic component (Figure 8i). This observation, in conjunction with calculated $\mathrm{PBE}+\mathrm{TS}$ energy differences, strongly suggests that $\mathrm{X}^{+} \mathrm{X}^{+}$(or $\mathrm{X}^{+} \mathrm{X}^{-}$) is the most likely structure to be found within $[\mathrm{AE} 2 \mathrm{~T}] \mathrm{PbI}_{4}$. The remaining structures all reflect a picture where the inorganic HOMO energy is lower than the organic HOMO energy, corresponding to a quasi-type-IIb alignment (Figure 8j) that allows for charge separation of carriers across the organic-inorganic interface (i.e., likely leading to quenching of the PL) in contrast with the observed excitonic PL. From the energy difference between inorganic and organic HOMO levels across all the panels in Figure 8, we find that the relative energy level alignment at the VBM can vary from $-0.11 \mathrm{eV}(\mathrm{LL})$ to $0.16 \mathrm{eV}\left(\mathrm{X}^{+} \mathrm{X}^{-}\right)$as a result of different AE2T orientation patterns. The significant variation $(0.27 \mathrm{eV})$ of relative energy alignment due to the local molecular arrangement in different structure models changes the qualitative character of the VBM in Figures 7 and 8. This demonstrates the importance of resolving the local structural correlation between adjacent molecules, which is critical in order to obtain the correct energy level alignment and QW character for these oligothiophene-based HOIPs.

So far, the relative configuration of the inorganic layers has remained fixed for all the above structural models. To definitively validate our hypothesis that a single configuration of the inorganic layers (with respect to Figure 3) can be used without any loss of generality, we have considered a model where the two organic layers are fixed in the $\mathrm{X}^{+} \mathrm{X}^{+}$ configuration, but the inorganic layer configuration is switched in a way that the upper inorganic layer remains the same, but the middle inorganic layer has an opposite square distortion (see Figure S2). The optimized lattice parameters (see Table $1)$, PBE total energy $(\Delta E=0.002 \mathrm{meV} /$ atom $)$, and DFTHSE06 + SOC band structures (Figure S3) are very similar for both the corresponding structures, suggesting that they are equivalent in energy and that the adjacent inorganic layers have the freedom to rotate with respect to one another, contributing to the observed RSD in $[\mathrm{AE} 2 \mathrm{~T}] \mathrm{PbI}_{4}$.

\section{CONCLUSIONS}

In conclusion, RSD can arise in 2D HOIPs when the distortion pattern in the metal halide framework can adopt two different (related by rotation) configurations that are energetically equivalent and when the organic tethering groups are flexible enough to allow (through hydrogen bonding) access to each of these configurations for each perovskite layer-that is, the organic cations also contribute to the RSD. When these conditions are met, the inorganic framework, which typically plays the most substantial role in scattering X-rays compared to the organic structural component, can quasi-randomly adopt one or the other of the configurations, giving rise to RSD and $\mathrm{DXS}$, as shown here for $[\mathrm{AE} 2 \mathrm{~T}] \mathrm{PbI}_{4}$. The observed XRD pattern of the latter could only be modeled crystallographically using a disordered $C 2 / c$ subcell because of the poorly resolved superstructure-related reflections (i.e., corresponding to not well-defined periodicity) along the stacking direction.
Considering the $\sim 30 \AA$ long-axis periodicity (i.e., corresponding to the local ordering) observed in the diffraction pattern of $[\mathrm{AE} 2 \mathrm{~T}] \mathrm{PbI}_{4}$, there are two unique inorganic perovskite layers (offset by lattice $C$-centering in the $C 2 / c$ average structure) within the enumerated prospective ordered supercells, each with two possible orientations corresponding to the two distortion patterns. The resulting four different inorganic layer configurations are all related by local symmetries and must be energetically equivalent, as also indicated by our DFT-PBE + TS energy calculations. The enumerated supercells also have various ordered AE2T configurations ranging between $\beta$-type and herringbone-type packing arrangements, both of which have been separately observed among oligothiophene-containing 2D HOIPs. The total energies and VBM energy offsets $E_{\text {(inorg. Номо) }}-$ $E_{\text {(org. HOMO) }}$ are found to vary considerably depending on the AE2T configuration, leading to either type-Ib or type-IIb heterostructure band alignments. The two most stable structures of $[\mathrm{AE} 2 \mathrm{~T}] \mathrm{PbI}_{4}$ found here also exhibit two energetically equivalent herringbone configurations of AE2T, which are denoted as $\mathrm{X}^{+} \mathrm{X}^{+}$and $\mathrm{X}^{+} \mathrm{X}^{-}$. The structurally equivalent inorganic, as well as the energetically equivalent organic configurations, can occur randomly with evidently no preference for a specific ordering, thereby inducing RSD.

Importantly, understanding the preferred local structural arrangement matters significantly for the predicted electronic properties. In the present example, the locally preferred molecular arrangement determines the details of the predicted electronic structure, making the difference between the observed type-Ib alignment and the alternative, a possible type-IIb band alignment that is not, in actuality, observed (as inferred from optical measurements). In general, 2D perovskites offer great potential as rationally tunable semiconductors through appropriate selection of the organic versus the inorganic components. The present paper shows that accurately understanding the details of the structure with its potentially many degrees of freedom (including RSD) is a key prerequisite for a reliable a priori prediction of properties.

\section{ASSOCIATED CONTENT}

\section{S Supporting Information}

The Supporting Information is available free of charge on the ACS Publications website at DOI: 10.1021/acs.chemmater.9b03208.

$298 \mathrm{~K}$ single-crystal data; schematic representation of hydrogen bonding interactions in $[\mathrm{AE} 2 \mathrm{~T}] \mathrm{PbI}_{4}$; two possible inorganic configurations used for band structure calculations; electronic band structure of the $\mathrm{X}^{+} \mathrm{X}^{+}$ structure model employing a different inorganic configuration; comparison of $\mathrm{X}^{+} \mathrm{X}^{-}$band structures computed using FHI-aims' "intermediate" and "tight" numerical settings; and input and relaxed geometry.in files of various structural models (PDF) Crystallographic data for $[\mathrm{AE} 2 \mathrm{~T}] \mathrm{PbI}_{4}$ at $298 \mathrm{~K}$ (CIF)

\section{AUTHOR INFORMATION}

\section{Corresponding Author}

*E-mail: david.mitzi@duke.edu. ORCID ${ }^{\circ}$

Sven Lidin: 0000-0001-9057-8233

Volker Blum: 0000-0001-8660-7230

David B. Mitzi: 0000-0001-5189-4612 


\section{Notes}

The authors declare no competing financial interest.

\section{ACKNOWLEDGMENTS}

M.K.J., C.L., D.J.D., W.Y., V.B., and D.B.M. acknowledge funding from the NSF DMREF program $\left(\mathrm{HybriD}^{3}\right)$ under grant numbers DMR-1729297 and DMR-1728921. S.L. acknowledges financial support from the Swedish Nation Research council. An award of computer time was provided by the INCITE program. This research used resources of the Argonne Leadership Computing Facility, which is a DOE Office of Science User Facility supported under Contract DEAC02-06CH11357.

\section{REFERENCES}

(1) Kagan, C. R.; Mitzi, D. B.; Dimitrakopoulos, C. D. OrganicInorganic Hybrid Materials as Semiconducting Channels in Thin-Film Field-Effect Transistors. Science 1999, 286, 945.

(2) Mitzi, D. B.; Dimitrakopoulos, C. D.; Rosner, J.; Medeiros, D. R.; $\mathrm{Xu}, \mathrm{Z}$.; Noyan, C. Hybrid Field-Effect Transistor Based on a LowTemperature Melt-Processed Channel Layer. Adv. Mater. 2002, 14, $1772-1776$.

(3) Smith, I. C.; Hoke, E. T.; Solis-Ibarra, D.; McGehee, M. D.; Karunadasa, H. I. A Layered Hybrid Perovskite Solar-Cell Absorber with Enhanced Moisture Stability. Angew. Chem. 2014, 126, 1141411417.

(4) Tsai, H.; Nie, W.; Blancon, J.-C.; Stoumpos, C. C.; Asadpour, R.; Harutyunyan, B.; Neukirch, A. J.; Verduzco, R.; Crochet, J. J.; Tretiak, $S$.; et al. High-efficiency two-dimensional Ruddlesden-Popper perovskite solar cells. Nature 2016, 536, 312.

(5) Van Le, Q.; Jang, H. W.; Kim, S. Y. Recent Advances toward High-Efficiency Halide Perovskite Light-Emitting Diodes: Review and Perspective. Small Methods 2018, 2, 1700419.

(6) Yin, J.; Maity, P.; Xu, L.; El-Zohry, A. M.; Li, H.; Bakr, O. M.; Brédas, J.-L.; Mohammed, O. F. Layer-Dependent Rashba Band Splitting in 2D Hybrid Perovskites. Chem. Mater. 2018, 30, 85388545.

(7) Zhai, Y.; Baniya, S.; Zhang, C.; Li, J.; Haney, P.; Sheng, C.-X.; Ehrenfreund, E.; Vardeny, Z. V. Giant Rashba splitting in 2D organicinorganic halide perovskites measured by transient spectroscopies. Sci. Adv. 2017, 3, No. e1700704.

(8) Zhao, B.; Bai, S.; Kim, V.; Lamboll, R.; Shivanna, R.; Auras, F.; Richter, J. M.; Yang, L.; Dai, L.; Alsari, M.; et al. High-efficiency perovskite-polymer bulk heterostructure light-emitting diodes. Nat. Photonics 2018, 12, 783-789.

(9) Zhao, X.; Ng, J. D. A.; Friend, R. H.; Tan, Z.-K. Opportunities and Challenges in Perovskite Light-Emitting Devices. ACS Photonics 2018, 5, 3866-3875.

(10) Saparov, B.; Mitzi, D. B. Organic-Inorganic Perovskites: Structural Versatility for Functional Materials Design. Chem. Rev. 2016, 116, 4558-4596.

(11) Hong, X.; Ishihara, T.; Nurmikko, A. V. Photoconductivity and electroluminescence in lead iodide based natural quantum well structures. Solid State Commun. 1992, 84, 657-661.

(12) Mitzi, D. B.; Chondroudis, K.; Kagan, C. R. Organic-inorganic electronics. IBM J. Res. Dev. 2001, 45, 29-45.

(13) Hu, J.; Oswald, I. W. H.; Hu, H.; Stuard, S. J.; Nahid, M. M.; Yan, L.; Chen, Z.; Ade, H.; Neilson, J. R.; You, W. Aryl-Perfluoroaryl Interaction in Two-Dimensional Organic-Inorganic Hybrid Perovskites Boosts Stability and Photovoltaic Efficiency. ACS Mater. Lett. 2019, 1, 171-176.

(14) Hu, J.; Oswald, I. W. H.; Stuard, S. J.; Nahid, M. M.; Zhou, N.; Williams, O. F.; Guo, Z.; Yan, L.; Hu, H.; Chen, Z.; et al. Synthetic control over orientational degeneracy of spacer cations enhances solar cell efficiency in two-dimensional perovskites. Nat. Commun. 2019, 10, 1276.
(15) Liu, C.; Huhn, W.; Du, K.-Z.; Vazquez-Mayagoitia, A.; Dirkes, D.; You, W.; Kanai, Y.; Mitzi, D. B.; Blum, V. Tunable Semiconductors: Control over Carrier States and Excitations in Layered Hybrid Organic-Inorganic Perovskites. Phys. Rev. Lett. 2018, 121, 146401.

(16) Hong, X.; Ishihara, T.; Nurmikko, A. V. Dielectric confinement effect on excitons inPbI4-based layered semiconductors. Phys. Rev. B: Condens. Matter Mater. Phys. 1992, 45, 6961-6964.

(17) Blancon, J.-C.; Stier, A. V.; Tsai, H.; Nie, W.; Stoumpos, C. C.; Traoré, B.; Pedesseau, L.; Kepenekian, M.; Katsutani, F.; Noe, G. T.; et al. Scaling law for excitons in $2 \mathrm{D}$ perovskite quantum wells. Nat. Commun. 2018, 9, 2254.

(18) Smith, M. D.; Connor, B. A.; Karunadasa, H. I. Tuning the Luminescence of Layered Halide Perovskites. Chem. Rev. 2019, 119, 3104-3139.

(19) Liang, C.; Zhao, D.; Li, Y.; Li, X.; Peng, S.; Shao, G.; Xing, G. Ruddlesden-Popper Perovskite for Stable Solar Cells. Energy Environ. Mater. 2018, 1, 221-231.

(20) Manser, J. S.; Christians, J. A.; Kamat, P. V. Intriguing Optoelectronic Properties of Metal Halide Perovskites. Chem. Rev. 2016, 116, 12956-13008.

(21) Thouin, F.; Valverde-Chávez, D. A.; Quarti, C.; Cortecchia, D.; Bargigia, I.; Beljonne, D.; Petrozza, A.; Silva, C.; Srimath Kandada, A. R. Phonon coherences reveal the polaronic character of excitons in two-dimensional lead halide perovskites. Nat. Mater. 2019, 18, 349356.

(22) Maheshwari, S.; Savenije, T. J.; Renaud, N.; Grozema, F. C. Computational Design of Two-Dimensional Perovskites with Functional Organic Cations. J. Phys. Chem. C 2018, 122, 17118-17122.

(23) Mitzi, D. B.; Chondroudis, K.; Kagan, C. R. Design, Structure, and Optical Properties of Organic-Inorganic Perovskites Containing an Oligothiophene Chromophore. Inorg. Chem. 1999, 38, 6246-6256.

(24) Zhang, S.-F.; Chen, X.-K.; Ren, A.-M.; Li, H.; Bredas, J.-L. Impact of Organic Spacers on the Carrier Dynamics in 2D Hybrid Lead-Halide Perovskites. ACS Energy Lett. 2019, 4, 17-25.

(25) Ema, K.; Inomata, M.; Kato, Y.; Kunugita, H.; Era, M. Nearly Perfect Triplet-Triplet Energy Transfer from Wannier Excitons to Naphthalene in Organic-Inorganic Hybrid Quantum-Well Materials. Phys. Rev. Lett. 2008, 100, 257401.

(26) Hu, H.; Meier, F.; Zhao, D.; Abe, Y.; Gao, Y.; Chen, B.; Salim, T.; Chia, E. E. M.; Qiao, X.; Deibel, C.; et al. Efficient RoomTemperature Phosphorescence from Organic-Inorganic Hybrid Perovskites by Molecular Engineering. Adv. Mater. 2018, 30, 1707621.

(27) Passarelli, J. V.; Fairfield, D. J.; Sather, N. A.; Hendricks, M. P.; Sai, H.; Stern, C. L.; Stupp, S. I. Enhanced Out-of-Plane Conductivity and Photovoltaic Performance in $\mathrm{n}=1$ Layered Perovskites through Organic Cation Design. J. Am. Chem. Soc. 2018, 140, 7313-7323.

(28) Gao, P.; Bin Mohd Yusoff, A. R.; Nazeeruddin, M. K. Dimensionality engineering of hybrid halide perovskite light absorbers. Nat. Commun. 2018, 9, 5028.

(29) Mao, L.; Stoumpos, C. C.; Kanatzidis, M. G. Two-Dimensional Hybrid Halide Perovskites: Principles and Promises. J. Am. Chem. Soc. 2018, 141, 1171-1190.

(30) Mitzi, D. B. Templating and structural engineering in organicinorganic perovskites. J. Chem. Soc., Dalton Trans. 2001, 1-12.

(31) Mitzi, D. B. Synthesis, Structure, and Properties of OrganicInorganic Perovskites and Related Materials; Progress in Inorganic Chemistry; Wiley, 1999; Vol. 48, pp 1-121.

(32) Smith, M. D.; Karunadasa, H. I. White-Light Emission from Layered Halide Perovskites. Acc. Chem. Res. 1999, 51, 619-627.

(33) Knutson, J. L.; Martin, J. D.; Mitzi, D. B. Tuning the Band Gap in Hybrid Tin Iodide Perovskite Semiconductors Using Structural Templating. Inorg. Chem. 2005, 44, 4699-4705.

(34) Chapuis, G.; Kind, R.; Arend, H. X-ray study of structural phase transitions in the perovskite-type layer compound $\left(\mathrm{CH}_{3} \mathrm{NH}_{3}\right)_{2} \mathrm{CdCl}_{4}$. Phys. Status Solidi A 1976, 36, 285-295.

(35) Mitzi, D. B.; Dimitrakopoulos, C. D.; Kosbar, L. L. Structurally Tailored Organic-Inorganic Perovskites: Optical Properties and 
Solution-Processed Channel Materials for Thin-Film Transistors. Chem. Mater. 2001, 13, 3728-3740.

(36) Mitzi, D. B. Organic-Inorganic Perovskites Containing Trivalent Metal Halide Layers: The Templating Influence of the Organic Cation Layer. Inorg. Chem. 2000, 39, 6107-6113.

(37) Jana, M. K.; Janke, S. M.; Dirkes, D. J.; Dovletgeldi, S.; Liu, C.; Qin, X.; Gundogdu, K.; You, W.; Blum, V.; Mitzi, D. B. DirectBandgap 2D Silver-Bismuth Iodide Double Perovskite: The StructureDirecting Influence of an Oligothiophene Spacer Cation. J. Am. Chem. Soc. 2019, 141, 7955-7964.

(38) Dornberger-Schiff, K.; Fichtner, K. On the Symmetry of ODStructures Consisting of Equivalent Layers. Krist. Tech. 1972, 7, $1035-1056$

(39) Li, Z.; Yan, X.; Tang, Z.; Huo, Z.; Li, G.; Jiao, L.; Liu, L.-M.; Zhang, M.; Luo, J.; Zhu, J. Direct observation of multiple rotational stacking faults coexisting in freestanding bilayer $\mathrm{MoS}_{2}$. Sci. Rep. 2017, $7,8323$.

(40) Warner, J. H.; Rümmeli, M. H.; Gemming, T.; Büchner, B.; Briggs, G. A. D. Direct Imaging of Rotational Stacking Faults in Few Layer Graphene. Nano Lett. 2009, 9, 102-106.

(41) Fichtner, K. Order-disorder structures. Comput. Math. Appl. 1988, 16, 469-477.

(42) Lebedev, A.; Tairov, Y. Polytypism in SiC: Theory and experiment. J. Cryst. Growth 2014, 401, 392-396.

(43) Dunlap-Shohl, W. A.; Barraza, E. T.; Barrette, A.; Dovletgeldi, S.; Findik, G.; Dirkes, D. J.; Liu, C.; Jana, M. K.; Blum, V.; You, W.; et al. Tunable internal quantum well alignment in rationally designed oligomer-based perovskite films deposited by resonant infrared matrix-assisted pulsed laser evaporation. Mater. Horiz. 2019, 6, 1707-1716.

(44) Dolomanov, O. V.; Bourhis, L. J.; Gildea, R. J.; Howard, J. A. K.; Puschmann, H. OLEX2: A complete structure solution, refinement and analysis program. J. Appl. Crystallogr. 2009, 42, 339-341.

(45) Ihrig, A. C.; Wieferink, J.; Zhang, I. Y.; Ropo, M.; Ren, X.; Rinke, P.; Scheffler, M.; Blum, V. Accurate localized resolution of identity approach for linear-scaling hybrid density functionals and for many-body perturbation theory. New J. Phys. 2015, 17, 093020.

(46) Blum, V.; Gehrke, R.; Hanke, F.; Havu, P.; Havu, V.; Ren, X.; Reuter, K.; Scheffler, M. Ab initio molecular simulations with numeric atom-centered orbitals. Comput. Phys. Commun. 2009, 180, 21752196.

(47) Havu, V.; Blum, V.; Havu, P.; Scheffler, M. Efficient integration for all-electron electronic structure calculation using numeric basis functions. J. Comput. Phys. 2009, 228, 8367-8379.

(48) Levchenko, S. V.; Ren, X.; Wieferink, J.; Johanni, R.; Rinke, P.; Blum, V.; Scheffler, M. Hybrid functionals for large periodic systems in an all-electron, numeric atom-centered basis framework. Comput. Phys. Commun. 2015, 192, 60-69.

(49) Ren, X.; Patrick, R.; Volker, B.; Jürgen, W.; Alexandre, T.; Andrea, S.; Karsten, R.; Matthias, S. Resolution-of-identity approach to Hartree-Fock, hybrid density functionals, RPA, MP2 and GW with numeric atom-centered orbital basis functions. New J. Phys. 2012, 14, 053020 .

(50) Yu, V. W.-z.; Corsetti, F.; García, A.; Huhn, W. P.; Jacquelin, M.; Jia, W.; Lange, B.; Lin, L.; Lu, J.; Mi, W.; et al. ELSI: A unified software interface for Kohn-Sham electronic structure solvers. Comput. Phys. Commun. 2018, 222, 267-285.

(51) Marek, A.; Blum, V.; Johanni, R.; Havu, V.; Lang, B.; Auckenthaler, T.; Heinecke, A.; Bungartz, H.-J.; Lederer, H. The ELPA library: scalable parallel eigenvalue solutions for electronic structure theory and computational science. J. Phys.: Condens. Matter 2014, 26, 213201.

(52) Perdew, J. P.; Burke, K.; Ernzerhof, M. Generalized Gradient Approximation Made Simple. Phys. Rev. Lett. 1996, 77, 3865-3868.

(53) Tkatchenko, A.; Scheffler, M. Accurate Molecular Van Der Waals Interactions from Ground-State Electron Density and FreeAtom Reference Data. Phys. Rev. Lett. 2009, 102, 073005.
(54) Heyd, J.; Scuseria, G. E.; Ernzerhof, M. Hybrid functionals based on a screened Coulomb potential. J. Chem. Phys. 2003, 118, 8207; J. Chem. Phys. 2006, 124, 219906.

(55) Huhn, W. P.; Blum, V. One-hundred-three compound bandstructure benchmark of post-self-consistent spin-orbit coupling treatments in density functional theory. Phys. Rev. Mater. 2017, 1, 033803.

(56) Chaloner, P. A.; Gunatunga, S. R.; Hitchcock, P. B. Redetermination of 2,2'-bithiophene. Acta Crystallogr., Sect. C: Cryst. Struct. Commun. 1994, 50, 1941-1942.

(57) Zhu, X.-H.; Mercier, N.; Frère, P.; Blanchard, P.; Roncali, J.; Allain, M.; Pasquier, C.; Riou, A. Effect of Mono- versus Diammonium Cation of $2,2^{\prime}$-Bithiophene Derivatives on the Structure of Organic-Inorganic Hybrid Materials Based on Iodo Metallates. Inorg. Chem. 2003, 42, 5330-5339.

(58) Fichou, D. Structural order in conjugated oligothiophenes and its implications on opto-electronic devices. J. Mater. Chem. 2000, 10, 571-588. 